\title{
COMO MEDIR A COMPETITIVIDADE DAS ORGANIZAÇÕES
}

\section{HOW TO EVALUATE THE COMPETITIVENESS OF THE ORGANIZATIONS}

\author{
José Ivan Alvares Xavier Ferreira \\ Engenheiro Mecânico \\ Doutor em Engenharia Mecânica \\ Departamento de Gestão Empresarial \\ CenPRA - Centro de Pesquisas Renato Archer \\ Rod. D. Pedro I, km 143,6, CEP 13082-120, Campinas - SP \\ Tel.: (19) 3746-6127Ｅ-mail: ivan.ferreira@cenpra.gov.br
}

\section{RESUMO}

Este trabalho trata do desenvolvimento de um modelo para aferir a competitividade das organizações focalizando os aspectos relacionados com: parâmetros organizacionais, sistemas de informação, medidas de desempenho e padrões de competitividade. Estabelecem-se relações de causa e efeito ponderadas entre os elementos destes componentes, através do uso de matrizes de correlação e obtém-se o modelo de referência. Discute-se a aplicação do modelo em empresas através do uso de um questionário e como a partir das respostas ao questionário aplicam-se os dados nas matrizes de correlação buscando a relação entre os diversos componentes do modelo. Aplica-se o modelo em quatro empresas, determinando-se o grau (estado) de competitividade delas tendo como base de comparação o modelo de referência. Desta forma, torna-se possível determinar como cada empresa localiza-se, com relação ao seu grau de competitividade, e onde ela poderia estar se utilizasse meios adequados.

Palavras-chave: competitividade, organização de aprendizagem, sistemas de informação, medidas de desempenho 
This work deals with the development of a model to evaluate the competitiveness of the organizations. The model focuses on organizational parameters, information systems, performance measures, and standards of competitiveness. Cause and effect relationships are established and pondered among the elements of these components. Through the use of correlation matrices a reference model is obtained. The model is applied in organizations through the use of a questionnaire, which provides the necessary correlation data in a matrix format that establishes the relations among various components of the model. The model is applied in four companies, determining the degree (state) of "competitiveness" among them. The model proved useful to "place" companies in relationship to its degree of competitiveness, and where it could be if it had used appropriate means

Key-words: competitiveness, learning organization, information systems, performance measures.

\section{INTRODUÇÃO}

O aparecimento de novas tecnologias, o desenvolvimento dos recursos da Tecnologia da Informação e o crescimento das telecomunicações, dentre outros fatos, contribuíram para o aparecimento de uma nova ordem mundial conhecida como globalização da economia. A tendência é que cada vez mais as empresas deixem de ser apenas locais e passem a competir no mercado internacional. A concorrência no mercado interno ou externo exige que as empresas sejam mais competitivas (PORTER, 2000). Com o intuito de determinar o estado de competitividade em que se encontram as empresas, propõe-se neste trabalho um modelo para aferi-la. Desta forma, na seção 2 discute-se a estrutura do modelo e seus componentes. $\mathrm{Na}$ seção 3 discute-se o modelo de referência e o método para obtê-lo. A seção 4 aborda a aplicação do modelo em 4 empresas possibilitando medir de forma quantitativa o estado (grau) de competitividade de cada uma delas através da utilização do nível de aderência e a ocorrência de aderência de configuração.

\section{ESTRUTURA DO MODELO}

A estrutura do modelo proposto é constituída dos componentes: parâmetros organizacionais (com características das organizações que aprendem), sistemas de informação, medidas de desempenho e padrões de competitividade, conforme mostra Figura 1 (FERREIRA, 2002). 


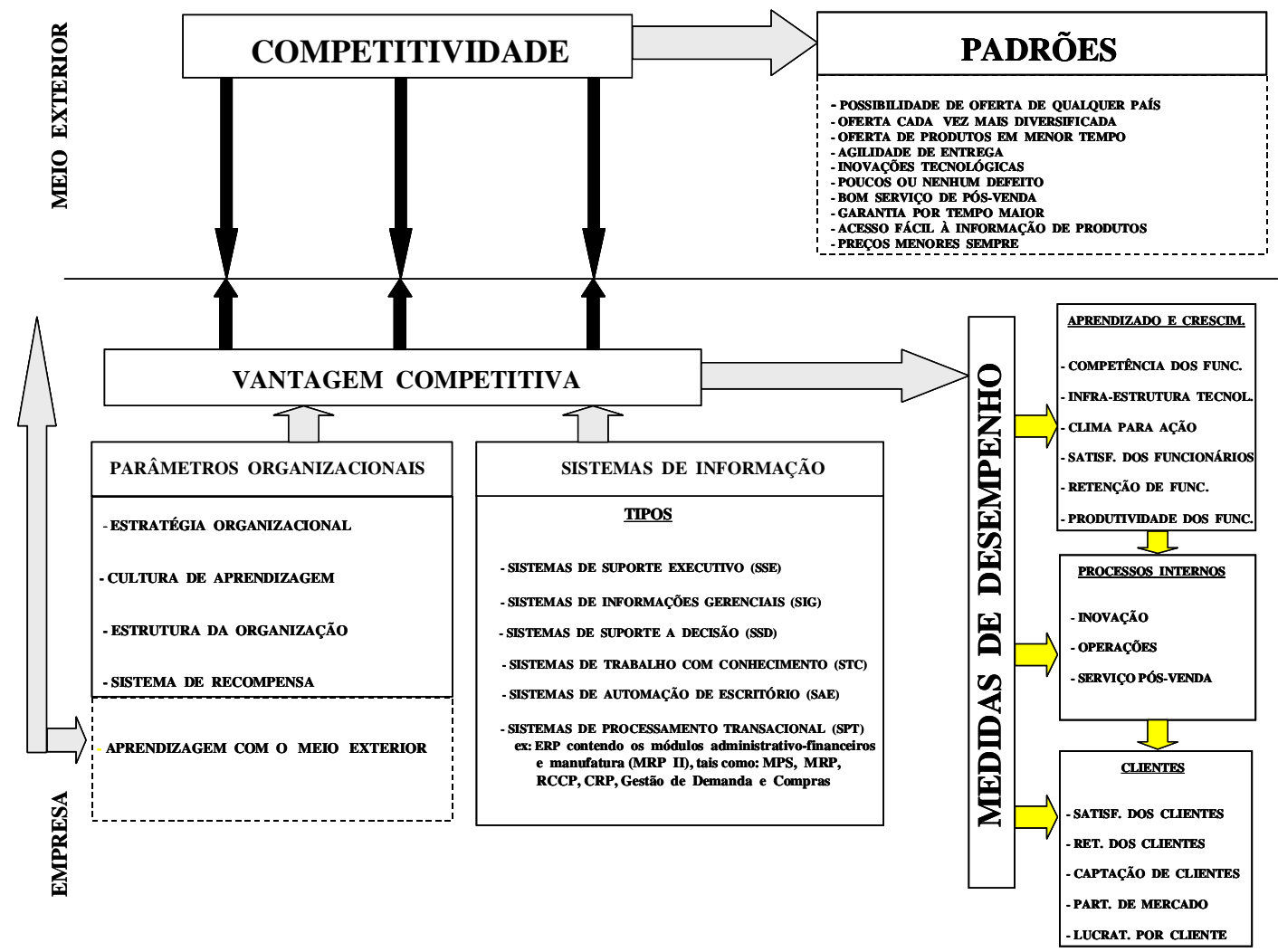

Figura 1: Estrutura do modelo de correlação

Fonte: FERREIRA, José I.A.X. (2002, p. 72)

Inicialmente define-se competitividade como a capacidade de uma organização de oferecer ao mercado alternativas capazes de motivar a troca da organização detentora de produto para aquela substituta (AGOSTINHO, 2001). Desta forma, é o cliente quem escolhe o fornecedor. Este deve atender às necessidades e expectativas do cliente (comprador) melhor do que seu competidor.

A competitividade está presente no meio exterior à empresa tanto em nível local como regional, nacional e global. Ela é verificada pelos padrões de competitividade.

Estes padrões são caracterizados por: (1) possibilidade de oferta de qualquer país; (2) oferta cada vez mais diversificada; (3) oferta de produtos em menor tempo; (4) agilidade de entrega; (5) inovações tecnológicas; (6) poucos ou nenhum defeito; (7) bom serviço de pósvenda; (8) garantia por tempo maior; (9) acesso fácil à informação de produtos; (10) preços menores sempre (AGOSTINHO, 2002).

Os padrões de competitividade variam de forma independente buscando atingir patamares que atendam cada vez mais as expectativas dos clientes que constituem o mercado consumidor. Os padrões de competitividade são detalhados no item 2.1. 
A competitividade, presente no meio exterior, exerce fortes pressões sobre a empresa. Esta deve reagir a essas pressões criando vantagem competitiva.

A empresa procura obter vantagem competitiva para satisfazer melhor os padrões de competitividade de uma maneira que o cliente não possa encontrar estas condições entre os competidores da empresa (FERREIRA, 2002).

A vantagem competitiva é, cada vez mais, função da competência com que uma empresa pode administrar e coordenar suas atividades. Desta forma, torna-se importante a existência de medidas da vantagem competitiva denominadas medidas de desempenho, que correspondem a medir a performance da organização. A obtenção da vantagem competitiva é expressa pelos valores numéricos das medidas de desempenho. As principais medidas de desempenho estão relacionadas com aprendizado e crescimento, processos internos e clientes, que por sua vez estão associados a: competência dos funcionários, infra-estrutura tecnológica, clima para ação, satisfação dos funcionários, retenção de funcionários, produtividade dos funcionários, inovação, operações, serviço pós-venda, satisfação dos clientes, retenção dos clientes, captação de clientes, participação de mercado e lucratividade por cliente. Estas medidas são consideradas no item 2.4.

A vantagem competitiva é obtida através dos parâmetros organizacionais que são constituídos por componentes associados às características das organizações que aprendem e criam conhecimento. Os elementos destes componentes são práticas gerenciais importantes e são considerados no item 2.2. Os principais componentes dos parâmetros organizacionais são: estratégia organizacional, cultura de aprendizagem, estrutura da organização, sistema de recompensa e aprendizagem com o meio exterior, considerados também no item 2.2.

A vantagem competitiva é obtida também através do alinhamento dos parâmetros organizacionais com os sistemas de informação. Isto significa que os diversos tipos de sistemas de informação exercem um papel importante na coordenação das atividades da empresa sem estar em conflito com os parâmetros organizacionais. Os tipos de sistemas de informação são considerados no item 2.3.

Portanto, a vantagem competitiva de uma organização é obtida através de vários fatores que, bem gerenciados, levam a organização a patamares de desempenho cada vez maiores. Isto tem como reflexo um aumento na participação de mercado e lucratividade.

Existe uma correlação entre competitividade externa e vantagem competitiva interna na medida em que a empresa procura satisfazer as necessidades dos clientes de forma melhor que seus competidores, mas mantendo uma performance que lhe garanta lucratividade. Desta 
forma, aumentando-se o ganho de vantagem competitiva aumenta-se a satisfação das necessidades dos clientes (compradores) de maneira a atender melhor os padrões de competitividade quando comparado com os competidores, mas mantendo um desempenho que garanta a lucratividade. Por sua vez, o ganho de vantagem competitiva aumenta na proporção em que os parâmetros organizacionais, compostos de práticas gerenciais, estejam sendo adotados e priorizados, além de estarem alinhados com os sistemas de informação. Por outro lado, as medidas da vantagem competitiva, expressas pelos valores numéricos das medidas de desempenho, atestam o ganho referido acima.

A Figura 1 apresentou o modelo de correlação entre a competitividade externa (verificada pelos padrões de competitividade), a vantagem competitiva interna à organização, as principais medidas da vantagem competitiva denominadas medidas de desempenho relacionadas com aprendizado e crescimento, processos internos e clientes (e associadas a: competência dos funcionários, infra-estrutura tecnológica, clima para ação, satisfação dos funcionários, retenção de funcionários, produtividade dos funcionários, inovação, operações, serviço pós-venda, satisfação dos clientes, retenção dos clientes, captação de clientes, participação de mercado, e lucratividade por cliente), os principais tipos de sistemas de informação (SSE, SIG, SSD, STC, SAE e SPT com ERP e MRP II, e seus principais módulos - MPS, MRP, RCCP, CRP, Gestão de Demanda e Compras), e os principais parâmetros organizacionais (estratégia organizacional, cultura de aprendizagem, estrutura da organização, sistema de recompensa, e aprendizagem com o meio exterior).

No próximo item são descritos os principais componentes do modelo de competitividade e suas principais inter-relações.

\subsection{Padrões de Competitividade}

Os padrões de competitividade devem ser vistos do ponto de vista dos mercados consumidores, mais especificamente na pessoa do comprador. Estes padrões expressam os desejos e expectativas dos compradores e criam a motivação necessária para que os consumidores saibam escolher de qual organização preferem utilizar um determinado produto. Deve-se considerar que é o comprador quem seleciona o fornecedor, sendo os padrões de competitividade que conduzem o comprador (cliente) a fazer a seleção.

Os padrões de competitividade são: 
Possibilidade de oferta de qualquer país: Constitui-se um fator de influência na escolha de um produto pelo cliente. Dentro de uma estratégia global, uma empresa pode vender em todos os países que representam mercados significativos para seus produtos. Para o consumidor isso representa a possibilidade de escolha de determinados produtos oriundos de vários países, ampliando assim, o leque de ofertas para sua decisão na compra.

Oferta cada vez mais diversificada: Significa maiores opções de escolha de determinados produtos pelo cliente. Este pode preferir o produto básico fornecido pela empresa ou o produto com vários graus de sofisticação, ou até mesmo o produto personalizado conforme sua vontade. Desta forma, a empresa que oferece maior diversificação de produtos tende a motivar mais o consumidor para o uso de seus produtos.

Oferta de produtos em menor tempo: Significa que o tempo de introdução de novos produtos no mercado, pela empresa, deva ser cada vez menor. Isto está relacionado com o tempo de resposta às necessidades do mercado, tendo importante influência na escolha de um produto de determinada empresa pelo cliente.

Agilidade de entrega: Caracteriza a rapidez com que um cliente é atendido pela empresa desde a solicitação de um produto até o seu respectivo recebimento. Isto implica muitas vezes em iniciar a fabricação do produto somente após a colocação do pedido de compra pelo cliente.

Inovações tecnológicas: Exercem significativa influência na decisão de escolha de um produto pelo consumidor. A inovação tecnológica é caracterizada como o desenvolvimento e introdução no mercado de um novo produto que incorpora uma nova tecnologia. Esse produto, com a nova tecnologia, gera importante atratividade para seu uso pelos clientes.

Poucos ou nenhum defeito: Significa a utilização de um produto pelo usuário, por muito tempo, sem apresentar defeitos. A satisfação que uma empresa proporciona a seu cliente, pelo uso de seu produto, comparado com o que a concorrência oferece, é conseguida durante a vida útil do produto, e não apenas na ocasião da compra.

Bom serviço pós-venda: É um padrão de competitividade que o cliente valoriza. A qualidade do serviço pós-venda intensifica e mantém o valor do produto. Isto inclui atividades junto ao cliente relacionadas com a instalação do produto, treinamento, eventuais reparos, ajustes do produto e fornecimento de peças, dentre outros.

Garantia por tempo maior: Significa na prática, para o usuário, um atestado de qualidade para o produto onde o fabricante garante por um determinado período de tempo que 
o produto não apresentará defeitos, desde que utilizado em condições normais de operação. Quanto maior for o tempo de garantia de um produto, maior será a motivação do cliente para adquiri-lo.

Acesso fácil à informação de produtos: Uma expectativa dos clientes antes de adquirirem um produto é ter facilidade para acessar as informações referentes a ele. Isto é importante para que o cliente saiba o que o produto faz, como ele opera, em que condições operam e outras informações mais.

Preços menores sempre: Constitui um padrão de competitividade que normalmente o cliente monitora antes de decidir pela compra do produto. Ao analisar o preço de um determinado produto, o cliente considera se é o preço que ele está realmente disposto a pagar, tendo como base os benefícios que este produto irá lhe proporcionar.

Finalmente, deve-se levar em conta que o comprador considera não apenas um único padrão de competitividade para decidir qual organização será fornecedora do produto que ele deseja. Ele considera vários daqueles padrões, priorizando-os conforme o critério que deseja, ou que irá melhor atender suas necessidades.

Por outro lado, as empresas criam vantagem competitiva interna para atender melhor as necessidades do comprador quando comparado com seus competidores.

Desta forma, os parâmetros organizacionais contribuem para a obtenção da vantagem competitiva na medida em que os elementos de seus componentes constituem práticas gerenciais importantes. Os parâmetros organizacionais são discutidos a seguir.

\subsection{Parâmetros Organizacionais}

Os parâmetros organizacionais são constituídos de componentes que estão associados às características das organizações que aprendem e criam conhecimento. BUCKLER (1998) define aprendizagem como "um processo que resulta na mudança de comportamento, de forma a obter-se melhoria de performance." Esta mudança de comportamento refere-se a três níveis: individual, equipe e organização.

Os componentes dos parâmetros organizacionais são: (1) estratégia organizacional; (2) cultura de aprendizagem; (3) estrutura da organização; (4) sistema de recompensa; (5) aprendizagem com o meio exterior (MCGILL \& SLOCUM, 2001).

Estratégia organizacional (EO): A alta administração exerce um papel fundamental neste contexto, pois é ela que estabelece as diretrizes estratégicas em busca da excelência da 
organização. As práticas gerenciais mais importantes consideradas neste tópico são: (1) compreensão, desenvolvimento e definição das competências essenciais da organização (PRAHALAD \& HAMEL, 2001); (2) divulgação da intenção estratégica da organização; (3) comunicação freqüente através de palestras, boletins e outros, para esclarecimento dos propósitos de mudanças com o intuito de engajar todos no processo de transformação da organização; (4) estabelecimento e estímulo ao atingimento de objetivos ambiciosos.

Cultura de aprendizagem (CA): Exerce um papel importante para promover a aprendizagem da organização. As seguintes práticas gerenciais são consideradas significativas: (1) incentivo ao aprendizado através de educação, treinamento e desenvolvimento, tais como programas, aulas e seminários; (2) presença na organização, de forma clara e consistente, das seguintes qualidades: abertura a experimentos; encorajamento para aceitar riscos, responsavelmente; e disposição de aceitar fracassos e aprender com eles (SENGE, 2002).

Estrutura organizacional (EstrO): As organizações que criam conhecimento possuem estruturas flexíveis e permeáveis. Estas organizações precisam encontrar maneiras criativas para conseguir melhorar a efetividade de todos seus processos operacionais e administrativos. Isto significa levar em conta que as melhorias radicais na efetividade envolvem fazer coisas de maneiras diferentes, e não somente fazê-las mais rápido (NADLER \& TUSHMAN, 1999). As práticas gerenciais mais relevantes são: (1) existência da permeabilidade nas fronteiras entre a gerência e os empregados; entre os departamentos funcionais; entre os empregados e clientes; e entre a empresa e seus vendedores, possibilitando maximizar o fluxo de informações, de idéias, de discernimento e de visões; (2) promoção da flexibilidade na estrutura para permitir adaptações e/ou criação de processos de negócio para atender as necessidades dos clientes; (3) utilização com freqüência de equipes autodirigidas dotadas de empowerment (FERREIRA, 1996).

Sistema de Recompensa (SR): Constitui uma característica importante dos parâmetros organizacionais no apoio à obtenção de vantagem competitiva. As práticas gerenciais relativas ao sistema de recompensa promovem: (1) incentivos através de premiação e reconhecimento dos funcionários por contribuições e resultados excelentes; (2) existência de formas de pagamento relacionado ao desempenho da equipe onde os créditos são distribuídos.

Aprendizagem com o meio exterior (AME): As organizações que aprendem com o meio exterior adotam práticas gerenciais onde existem: (1) procedimentos estabelecendo a busca de melhores práticas (realização de benchmarking) comparativamente a outras 
organizações; (2) realização de alianças estratégicas com objetivos claros de aprendizado importante.

As práticas gerenciais expostas neste item e que constituem os elementos dos componentes dos parâmetros organizacionais são responsáveis pela obtenção da vantagem competitiva. Além disso, elas devem estar alinhadas com os sistemas de informação. Estes são considerados a seguir.

\subsection{Sistemas de Informação}

Os sistemas de informação apoiam a obtenção de vantagem competitiva contribuindo, dentre outros aspectos, para a coordenação da empresa como um todo.

Ressalta-se também que quando há uma sinergia entre equipes e sistemas de informação, a contribuição destes dois para o conhecimento e desempenho organizacional é maior do que a soma das partes (MANKIN et all., 2002).

Segundo DRUCKER (1995), é importante também considerar a idéia que existe subjacente aos sistemas de informação. Essa idéia está freqüentemente associada a negócios, existindo uma interdependência e interatividade entre negócios e sistemas de informação.

Por outro lado, podem também ocorrer influências relacionadas com aspectos culturais. No Japão, por exemplo, a construção de sistemas não constitui um fim em si. Isso significa que a meta mais elevada é enaltecer a contribuição das pessoas. Desta forma, se um sistema automatiza um trabalho que as pessoas podem fazer melhor, esse não é considerado um bom sistema (BENSAOU \& EARL, 1998).

Os tipos de sistemas de informações considerados são: (1) sistemas de suporte executivo (SSE); (2) sistemas de informações gerenciais (SIG); (3) sistemas de suporte à decisão (SSD); (4) sistemas de trabalho com conhecimento (STC); (5) sistemas de automação de escritório (SAE); (6) sistemas de processamento transacional (STP) (LAUDON \& LAUDON, 2002).

O sistema de processamento transacional (STP) considerado é o ERP - Enterprise Resources Planning - constituído por seus módulos administrativo-financeiros e manufatura. Para a manufatura (sistemas MRP II) são considerados os seguintes módulos: (1) plano mestre de produção (MPS); (2) análise bruta de capacidade (RCCP); (3) planejamento das 
necessidades de material (MRP); (4) planejamento das necessidades de capacidade (CRP); (5) gestão de demanda; (6) compras (CORRÊA et all., 2002).

\subsection{Medidas de Desempenho}

A obtenção da vantagem competitiva é expressa pelos valores numéricos das medidas de desempenho. Desta forma, o modelo para aferição da competitividade considera medidas de desempenho relacionadas com as perspectivas: aprendizado e crescimento, processos internos e clientes (KAPLAN \& NORTON, 2001).

Aprendizado e crescimento: São consideradas as medidas ou indicadores, relacionados abaixo, relativos a: (1) competência dos funcionários, envolvendo conhecimento que inclui informação, experiência, contexto e reflexão (JOHNSTON \& BLUMENTRITT, 2000); (2) infra-estrutura tecnológica; (3) clima para ação; (4) satisfação dos funcionários; (5) retenção dos funcionários; (6) produtividade dos funcionários. Estas medidas expressam a obtenção da vantagem competitiva relacionada com aprendizado e crescimento.

Medida associada à competência dos funcionários: horas de treinamento por funcionário por ano, incluindo educação que é caracterizada pelos aspectos de conscientização.

Medida associada à infra-estrutura tecnológica: percentual de funcionários que lidam (trabalham) diretamente com os clientes e têm acesso on-line às informações referentes a eles.

Medidas associadas a clima para ação: (1) número de sugestões por funcionário; (2) percentual de equipes com incentivos compartilhados, caracterizado pela proporção de equipes em que os membros compartilham objetivos comuns; (3) percentual de projetos com incentivos individuais de equipes vinculados ao sucesso do projeto.

Medida associada à satisfação dos funcionários: pesquisa de satisfação dos funcionários.

Medida associada à retenção dos funcionários: percentual de rotatividade de pessoaschave.

Medida associada a produtividade dos funcionários: receita por funcionário.

Processos internos: São consideradas as medidas ou indicadores, relacionados abaixo, relativos a: inovação, operações, e serviço pós-venda. Estas medidas expressam a obtenção da vantagem competitiva relacionada com processos internos. 
Medidas associadas a inovação: (1) percentual de vendas gerados por novos produtos; (2) tempo de lançamento de um produto no mercado, caracterizado pelo tempo decorrido desde o início do desenvolvimento do produto, até o seu lançamento no mercado; (3) tempo de equilíbrio, caracterizado pelo tempo decorrido desde o início do desenvolvimento do produto até o seu lançamento e venda, gerando lucro suficiente para pagar o investimento originalmente feito em seu desenvolvimento.

Medidas associadas a operações: (1) tempo de ciclo, caracterizado como o tempo médio entre a colocação do pedido pelo cliente e a entrega do produto ao cliente; (2) giro de inventário anual, caracterizado como a relação percentual entre o valor do custo dos produtos vendidos (embarcados) no ano, pelo valor total do inventário. Esse inclui matéria prima, peças em processos e produtos acabados; (3) confiabilidade de entrega, caracterizada como a percentagem que expressa a quantidade dos pedidos solicitados pelos clientes que foram entregues nas datas prometidas para estes clientes; (4) índice de rejeição pelo cliente, caracterizado pelo número de unidades completas rejeitadas ou devolvidas pelos clientes, expresso em partes por milhão (ppm). O cálculo deve incluir também os componentes retrabalhados pelos clientes. Aplica-se a todas as unidades embarcadas, incluindo componentes.

Medidas associadas a serviço pós-venda: (1) rapidez de resposta aos defeitos, caracterizada como o tempo decorrido desde a solicitação do cliente até a resolução final do problema do cliente; (2) nível de atendimento imediato, caracterizado pelo percentual de solicitações dos clientes atendidos com uma única visita de serviços.

Clientes. São consideradas as medidas ou indicadores, relacionados abaixo, relativos a clientes. Estas medidas expressam a obtenção da vantagem competitiva relacionada com clientes.

Medidas associadas a clientes: (1) participação de mercado, caracterizada pela percentagem de vendas de produtos da empresa em relação ao total de vendas, num determinado mercado, de produtos similares; (2) captação de clientes, medida pelo número de novos clientes conquistados pela empresa no período (um ano); (3) retenção de clientes, medida pelo número de clientes atuais que continuam consumindo produtos da empresa no período (um ano); (4) satisfação dos clientes, medida pelo número de reclamações no período (um ano); (5) lucratividade por cliente, medida através do lucro líquido obtido por cliente ou segmento, deduzidas as despesas necessárias para sustentar esses clientes. 
Após ter-se discutido todos os elementos dos componentes do modelo de correlação conforme mostrado na Figura 1, discute-se a seguir o modelo de referência (reduzido).

\section{MODELO DE REFERÊNCIA REDUZIDO}

Com o objetivo de aferir a competitividade de empresas desenvolveu-se o modelo de referência reduzido (MRR) expresso através das Figuras 2 e 3 (FERREIRA, 2002). A Figura 2 corresponde ao Modelo de Referência Reduzido (MRR) com influência dos parâmetros organizacionais (PO); e a Figura 3 correspondente ao Modelo de Referência Reduzido (MRR) com influência dos sistemas de informação (SI). As Figuras 2 e 3 servirão de base para comparação posterior com as Figuras PO e SI obtidas a partir das respostas do questionário, para a coleta de dados, aplicado junto às empresas analisadas. Desta forma, quanto mais próximas estiverem as configurações das Figuras 2 e 3 das Figuras PO e SI da empresa analisada, respectivamente, maior será o seu grau (estado) de competitividade.

Apresenta-se a seguir, de forma resumida, os passos de 1 a 9 (FERREIRA, 2002) para se obter as Figuras 2 e 3.

1) Estabelecem-se as matrizes de correlação entre:

a) parâmetros organizacionais;

b) sistemas de informação;

com as respectivas medidas de desempenho (MD).

2) Estabelece-se a correlação ponderada, através da pontuação:

5 - forte correlação;

3 - média correlação;

1 - baixa correlação.

3) Ordena-se a importância dessas correlações, de forma numérica decrescente, nas colunas e linhas de resultado final.

4) Para melhor visualização na matriz, estabelecem-se cores, como segue:

escura (região A) - forte correlação - valor 5;

cinza (região B) - média correlação - valor 3;

clara (região C) - baixa correlação - valor 1.

Desta maneira, obtém-se análise visual do efeito ponderado.

Para o modelo de referência reduzido consideram-se as regiões mais importantes (A - forte correlação e B - média correlação). Desconsidera-se a região C - baixa correlação. 
Através dos passos anteriores 1,2,3 e 4, obtêm-se duas matrizes primárias ponderadas. Uma referente à correlação de PO com MD e outra de SI com MD.

5) Estabelece-se a mesma metodologia, para se estabelecer as correlações ponderadas entre:

a) parâmetros organizacionais;

b) sistemas de informação;

com os respectivos padrões de competitividade (PC), gerando-se outras duas matrizes primárias ponderadas, uma referente à correlação de PO com PC e outra de SI com PC.

6) A partir das correlações desenvolvidas: PO com MD citado no item 4 e PO com PC citado no item 5, gera-se a matriz de determinação da intensidade dos parâmetros organizacionais $(\mathrm{PO})$ sobre MD e PC onde se efetua a pontuação ponderada entre MD e PC e PO.

7) Ordena-se a importância dessas intensidades, de forma numérica decrescente, na coluna de resultado final.

8) A partir da determinação da intensidade dos parâmetros organizacionais (item 6) e sua ordenação (item 7), gera-se a Figura 2 que corresponde ao Modelo de Referência Reduzido (MRR) com influência dos parâmetros organizacionais (PO).

9) Estabelece-se a mesma metodologia a partir das correlações desenvolvidas: SI com MD citada no item 4 e SI com PC citada no item 5 para se gerar a Figura 3, que corresponde ao Modelo de Referência Reduzido (MRR) com influência dos sistemas de informação (SI). 


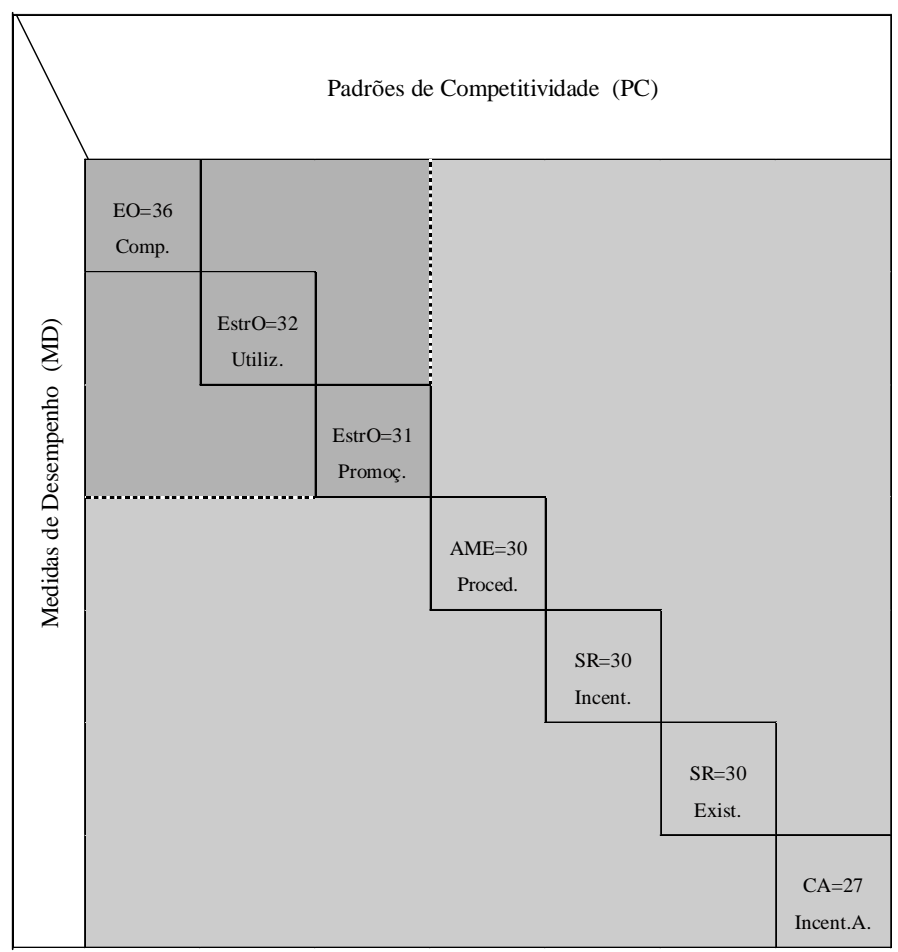

Figura 2: Modelo de referência reduzido com influência dos parâmetros organizacionais

Fonte: FERREIRA, José I.A.X. (2002,p. 150)

É importante notar na Figura 2 a seqüência das posições (de 1 a 7) dos elementos de PO. Eles estão ordenados segundo a diagonal da Figura 2, de forma descendente (do maior valor numérico para o menor). A seguir apresenta-se a lista referente à seqüência das posições dos elementos de PO. Nesta lista está indicada entre parênteses a abreviatura do componente de PO, o valor numérico correspondente à influência do elemento de PO listado e a abreviatura da primeira palavra do elemento de PO listado. Esta é também a forma utilizada para caracterizar o elemento de PO na Figura 2 em sua posição. Observa-se também que os elementos de PO na seqüência de 1, 2 e 3 situam-se na região ótima (região mais escura) e os elementos de PO na sequiência 4, 5, 6 e 7 situam-se na região boa (região mais clara). As regiões ótima e boa abrangem, respectivamente, de forma aproximada, $23 \%$ e $31 \%$ dos elementos de PO correspondente ao modelo de referência, que considera também a região marginal com $46 \%$ dos elementos de PO. A região marginal não faz parte do modelo de referência reduzido (MRR) mostrado na Figura 2.

1- Compreensão, desenvolvimento e definição das competências essenciais da organização (EO=36 Comp.);

2- Utilização com freqüência de equipes autodirigidas dotadas de empowerment (EstrO=32 Utiliz.); 
3- Promoção da flexibilidade na estrutura para permitir adaptações e/ou criação de processos de negócio para atender as necessidades dos clientes (EstrO=31 Promoç.);

4- Procedimentos estabelecendo a busca de melhores práticas (realização de benchmarking) comparativamente a outras organizações (AME=30 Proced.);

5- Incentivos através de premiação e reconhecimento dos funcionários por contribuições e resultados excelentes ( $\mathrm{SR}=30$ Incent.);

6- Existência de formas de pagamento relacionado ao desempenho da equipe onde os créditos são distribuídos ( $\mathrm{SR}=30$ Exist.);

7- Incentivo ao aprendizado através de educação, treinamento e desenvolvimento, tais como: programas, aulas e seminários ( $\mathrm{CA}=27$ Incent.A.).

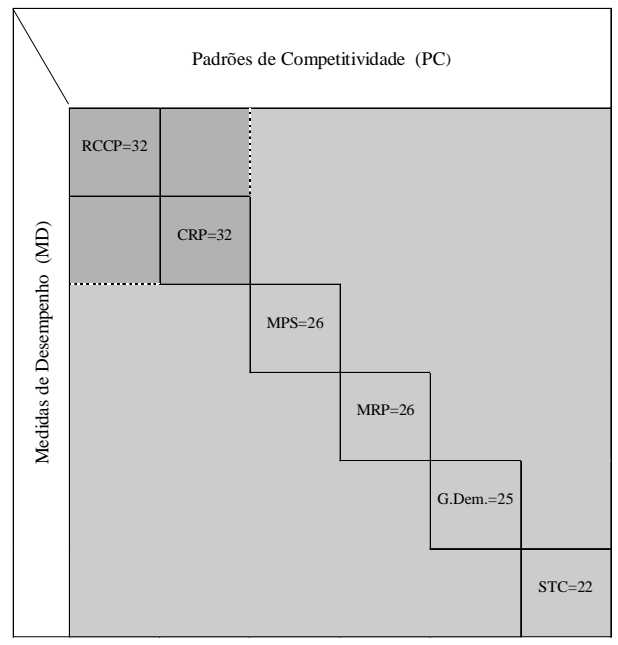

Figura 3: Modelo de referência reduzido com influência dos sistemas de informação

Fonte: FERREIRA, José I.A.X. (2002, p.154)

Ressalta-se também a importância na Figura 3 da sequiência das posições (de 1 a 6 ) dos componentes de SI. Eles estão ordenados segundo a diagonal da Figura 3, de forma descendente (do maior valor numérico para o menor). A seguir apresenta-se a lista referente à seqüência das posições dos componentes de SI. Nesta lista está indicada entre parênteses a abreviatura do componente de SI e o valor numérico correspondente à influência deste componente listado. Esta é também a forma utilizada para caracterizar o elemento de SI na Figura 3 em sua posição. Observa-se também que os componentes de SI na sequiência de 1 e 2 situam-se na região ótima (região mais escura) e os componentes de SI na seqüência 3, 4, 5 e 6 situam-se na região boa (região mais clara). As regiões ótima e boa abrangem, respectivamente, de forma aproximada, $17 \%$ e $33 \%$ dos componentes de SI correspondente ao modelo de referência, que considera também a região marginal com 50\% dos elementos de SI. 
A região marginal não faz parte do modelo de referência reduzido (MRR) mostrado na Figura 3.

1- Análise bruta de capacidade ( $\mathrm{RCCP}=32)$;

2- Planejamento das necessidades de capacidade ( $\mathrm{CRP}=32)$;

3- Plano mestre de produção (MPS=26);

4- Planejamento das necessidades de material $(\mathrm{MRP}=26)$;

5- Gestão de demanda (G.Dem.=25).

6- Sistemas de trabalho com conhecimento (STC=22).

\section{APLICAÇÃO}

O modelo de referência reduzido (MRR) utilizado para aferição da competitividade foi aplicado em quatro empresas do setor de telecomunicações situadas na região de Campinas (SP). Três delas (empresas A, B e C) possuem acima de 1100 funcionários e com receitas anuais acima de US\$ 200 milhões (cada uma delas). A quarta (empresa D) possui 375 funcionários com receita anual de US\$ 66 milhões.

Utilizou-se o questionário denominado "Avaliação do Grau de Correlação" (208 perguntas) para a coleta de dados junto às empresas analisadas. As pessoas que responderam o questionário (vice-presidente, diretores e gerentes) expressam uma opinião contextualizada no consenso porque detêm conhecimento. O questionário foi respondido por uma pessoa de cada empresa, com exceção da empresa $\underline{A}$ onde duas pessoas responderam o questionário. $\mathrm{O}$ respondente 1 (resp. 1) possui um perfil mais voltado para a área de produção e o respondente 2 (resp. 2) com perfil voltado mais para a área comercial. Ressalta-se também que para refletir a opinião da empresa, de forma mais exata, é necessário que várias pessoas chaves da empresa respondam o questionário ou que estas expressem um consenso para cada resposta do questionário.

Desta forma, cada empresa expressou o grau de intensidade da correlação entre os assuntos expressos nas frases do questionário assinalando uma das três alternativas: forte correlação (F), média correlação (M) e baixa correlação (B). A seguir apresenta-se exemplo do questionário (3 perguntas do total de 208).

a- O aumento da compreensão, desenvolvimento e definição das competências essenciais da organização: 
a1- contribuem para diminuir o tempo de lançamento de um novo produto no mercado.
F [ ]
M [ ]
$\mathrm{B}$ [ ]

a2- contribuem para aumentar a satisfação dos funcionários.
$\mathrm{F}[\mathrm{]}$
M [ ]
$\mathrm{B}[\mathrm{]}$

a3- contribuem para aumentar a receita por funcionário.
$\mathrm{F}[\mathrm{]}$
$\mathrm{M}[\mathrm{]}$
$\mathrm{B}$ [ ]

A partir das respostas do questionário elaboraram-se as Figuras PO e SI de cada empresa permitindo a elaboração das Figuras 4 e 5 (FERREIRA, 2002) que mostram, da esquerda para direita, a seqüência das posições dos elementos de PO e componentes de SI comparadas com as do modelo de referência reduzido, respectivamente.

\begin{tabular}{|c|c|c|c|c|c|c|c|}
\hline \multicolumn{8}{|c|}{ Seqüência das posições dos elementos de PO } \\
\hline MRR & $\begin{array}{l}\text { Comp. - } 1 \\
\text { (EO) }\end{array}$ & $\begin{array}{l}\text { Utiliz. - } 2 \\
\text { (EstrO) }\end{array}$ & $\begin{array}{c}\text { Promoç. - } 3 \\
\text { (EstrO) }\end{array}$ & $\begin{array}{l}\text { Proced. - } 4 \\
\text { (AME) }\end{array}$ & $\begin{array}{c}\text { Incent. - } 5 \\
\text { (SR) }\end{array}$ & $\begin{array}{c}\text { Exist. - } 6 \\
\text { (SR) }\end{array}$ & $\begin{array}{c}\text { Incent. A. - } 7 \\
\text { (CA) }\end{array}$ \\
\hline A - resp. 1 & $\begin{array}{c}\text { Incent.A. - } 7 \\
\text { (CA) }\end{array}$ & $\begin{array}{c}\text { Comp. - } 1 \\
\text { (EO) }\end{array}$ & $\begin{array}{l}\text { Promoç. - } 3 \\
\text { (EstrO) }\end{array}$ & $\begin{array}{l}\text { Utiliz. - } 2 \\
\text { (EstrO) }\end{array}$ & $\begin{array}{c}\text { Proced. - } 4 \\
\text { (AME) }\end{array}$ & $\begin{array}{c}\text { Exist. - } 6 \\
\text { (SR) }\end{array}$ & $\begin{array}{c}\text { Incent. - } 5 \\
\text { (SR) }\end{array}$ \\
\hline A - resp. 2 & $\begin{array}{c}\text { Comp. - } 1 \\
\text { (EO) }\end{array}$ & $\begin{array}{c}\text { Proced. - } 4 \\
\text { (AME) }\end{array}$ & $\begin{array}{l}\text { Utiliz. - } 2 \\
\text { (EstrO) }\end{array}$ & $\begin{array}{c}\text { Promoç. - } 3 \\
\text { (EstrO) }\end{array}$ & $\begin{array}{c}\text { Exist. - } 6 \\
\text { (SR) }\end{array}$ & $\begin{array}{c}\text { Incent. - } 5 \\
\text { (SR) }\end{array}$ & $\begin{array}{c}\text { Incent.A. - } 7 \\
\text { (CA) }\end{array}$ \\
\hline $\mathrm{B}$ & $\begin{array}{l}\text { Utiliz. - } 2 \\
\text { (EstrO) }\end{array}$ & $\begin{array}{c}\text { Proced. - } 4 \\
\text { (AME) }\end{array}$ & $\begin{array}{c}\text { Comp. - } 1 \\
\text { (EO) }\end{array}$ & $\begin{array}{c}\text { Promoç. - } 3 \\
\text { (EstrO) }\end{array}$ & $\begin{array}{c}\text { Exist. - } 6 \\
\text { (SR) }\end{array}$ & $\begin{array}{c}\text { Incent. - } 5 \\
\text { (SR) }\end{array}$ & $\begin{array}{c}\text { Incent.A. - } 7 \\
\text { (CA) }\end{array}$ \\
\hline $\mathrm{C}$ & $\begin{array}{c}\text { Promoç - } 3 \\
\text { (EstrO) }\end{array}$ & $\begin{array}{l}\text { Utiliz. - } 2 \\
\text { (EstrO) }\end{array}$ & $\begin{array}{l}\text { Proced. - } 4 \\
\text { (AME) }\end{array}$ & $\begin{array}{c}\text { Incent.A. - } 7 \\
\text { (CA) }\end{array}$ & $\begin{array}{c}\text { Comp. - } 1 \\
\text { (EO) }\end{array}$ & $\begin{array}{c}\text { Incent. - } 5 \\
\text { (SR) }\end{array}$ & $\begin{array}{c}\text { Exist. - } 6 \\
\text { (SR) }\end{array}$ \\
\hline $\mathrm{D}$ & $\begin{array}{l}\text { Utiliz. - 2 } \\
\text { (EstrO) }\end{array}$ & $\begin{array}{c}\text { Promoç. - } 3 \\
\text { (EstrO) }\end{array}$ & $\begin{array}{c}\text { Comp. - } 1 \\
\text { (EO) }\end{array}$ & $\begin{array}{c}\text { Incent.A. - } 7 \\
\text { (CA) }\end{array}$ & $\begin{array}{c}\text { Exist. - } 6 \\
\text { (SR) }\end{array}$ & $\begin{array}{c}\text { Incent. - } 5 \\
\text { (SR) }\end{array}$ & $\begin{array}{c}\text { Proced. - } 4 \\
\text { (AME) }\end{array}$ \\
\hline
\end{tabular}

Figura 4: Figura comparativa da seqüência das posições dos elementos de PO das empresas participantes com o modelo de referência reduzido (MRR)

Fonte: FERREIRA, José I.A.X. (2002, p. 169) 


\begin{tabular}{|c|c|c|c|c|c|c|}
\hline \multicolumn{7}{|c|}{ Sequiência das posições dos componentes de SI } \\
\hline MRR & RCCP - 1 & CRP - 2 & MPS - 3 & MRP - 4 & G.Dem. - 5 & STC - 6 \\
\hline A - resp. 1 & MRP - 4 & CRP - 2 & MPS - 3 & STC - 6 & G.Dem. - 5 & RCCP - 1 \\
\hline A - resp. 2 & STC - 6 & MRP - 4 & RCCP - 1 & G.Dem. - 5 & CRP - 2 & MPS - 3 \\
\hline $\mathrm{B}$ & RCCP - 1 & CRP - 2 & MPS - 3 & MRP - 4 & G.Dem. - 5 & STC - 6 \\
\hline $\mathrm{C}$ & RCCP - 1 & CRP - 2 & MPS - 3 & G.Dem. - 5 & MRP - 4 & STC - 6 \\
\hline D & STC - 6 & MPS - $\mathbf{3}$ & MRP - 4 & G.Dem. - 5 & RCCP - 1 & CRP - 2 \\
\hline
\end{tabular}

Figura 5: Figura comparativa da seqüência das posições dos componentes de SI das empresas participantes com o modelo de referência reduzido (MRR)

Fonte: FERREIRA, José I.A.X. (2002, p. 171)

\subsection{Análise de Aderência}

A análise de aderência consiste em efetuar um estudo para determinar o nível de aderência e a ocorrência ou não da aderência de configuração em relação ao modelo de referência reduzido (FERREIRA, 2002).

O nível de aderência tem por objetivo expressar o grau de competitividade das empresas participantes em termos percentuais, comparativamente ao MRR. Isto significa encontrar uma maneira que permita verificar qual a proximidade que existe entre a configuração das Figuras PO e SI das empresas participantes e o MRR, em termos quantitativos. Desta maneira, quanto maior a proximidade entre as duas configurações, maior é a aderência das Figuras PO e SI ao MRR. Como conseqüência, maior será o valor expresso em termos percentuais e mais competitiva é a empresa. Desta forma, pode-se também comparar a competitividade entre duas empresas tendo como base a aderência de suas Figuras PO e SI. ao MRR. Portanto, quanto maior o nível de aderência mais competitiva será a empresa.

A empresa será mais competitiva ainda se, além de possuir o nível de aderência máximo (100\%) em uma das regiões, possuírem também a ocorrência de aderência de configuração.

A configuração, tanto do MRR como das empresas participantes, é caracterizada pela seqüência das posições dos elementos de PO e dos componentes de SI mostrados nas Figuras 
4 e 5, respectivamente. A partir destas considerações passa-se às seguintes definições (FERREIRA, 2002):

Número de aderência na região ótima devido a PO - (Ndop): É o número de elementos de PO que a empresa participante possui nas três primeiras posições da sua seqüência, iguais aos do MRR situados na região ótima. Como exemplo, observando-se a Figura 4, o Ndop para a empresa A (respondente 1) é dois (2), pois os elementos Comp. e Promoç. desta empresa/respondente são iguais a dois dos elementos que estão situados na região ótima do MRR.

Número de aderência na região boa devido a PO - (Ndbp): É o número de elementos de PO que a empresa participante possui nas quatro últimas posições da sua seqüência, iguais aos do MRR situados na região boa. Como exemplo, observando-se a Figura 4, o Ndbp para a empresa A (respondente 1) é três (3), pois os elementos Proced., Exist. e Incent. desta empresa/participante são iguais a três dos elementos que estão situados na região boa do MRR.

Número de aderência na região ótima devido a SI - (Ndos): É o número de componentes de SI que a empresa participante possui nas duas primeiras posições da sua seqüência, iguais aos do MRR situados na região ótima. Como exemplo, observando-se a Figura 5, o Ndos para a empresa A (respondente 1) é um (1), pois o componente CRP desta empresa/respondente é igual a um dos componentes que estão situados na região ótima do MRR.

Número de aderência na região boa devido a SI - (Ndbs): É o número de componentes de SI que a empresa participante possui nas quatro últimas posições da sua sequiência, iguais aos do MRR situados na região boa. Como exemplo, observando-se a Figura 5, o $N d b s$ para a empresa A (respondente 1) é três (3), pois os componentes MPS, STC e G.Dem. desta empresa/respondente são iguais a três dos componentes que estão situados na região boa do MRR.

Nível de aderência na região ótima devido a PO - (Nvop): É a relação expressa em percentagem entre o Ndop e o número total de elementos de PO situados na região ótima do MRR, designado por Ntop. Desta maneira, tem-se:

Nvop $=($ Ndop/Ntop $) \times 100 \quad[\%]$

Como Ntop é sempre igual a três (3) conforme observar-se na Figura 4, obtém-se:

Nvop $=(\mathrm{Ndop} / 3) \times 100 \quad[\%]$ 
Nivel de aderência na região boa devido a PO - (Nvbp): É a relação expressa em percentagem entre o $N d b p$ e o número total de elementos de PO situados na região boa do MRR, designado por Ntbp. Desta maneira, tem-se:

$\mathrm{Nvbp}=(\mathrm{Ndbp} / \mathrm{Ntbp}) \times 100 \quad[\%]$

Como Ntbp é sempre igual a quatro (4) conforme observar-se na Figura 4, obtém-se:

$\mathrm{Nvbp}=(\mathrm{Ndbp} / 4) \times 100 \quad[\%]$

Nível de aderência na região ótima devido a SI - (Nvos): É a relação expressa em percentagem entre o Ndos e o número total de componentes de SI situados na região ótima do MRR, designado por Ntos. Desta maneira, tem-se:

Nvos $=($ Ndos/Ntos $) \times 100 \quad[\%]$

Como Ntos é sempre igual a dois (2) conforme observar-se na Figura 5, obtém-se:

Nvos $=(\mathrm{Ndos} / 2) \times 100 \quad[\%]$

Nível de aderência na região boa devido a $S I$ - (Nvbs): É a relação expressa em percentagem entre o Ndbs e o número total de componentes de SI situados na região boa do MRR, designado por Ntbs. Desta maneira, tem-se:

$\mathrm{Nvbs}=(\mathrm{Ndbs} / \mathrm{Ntbs}) \times 100 \quad[\%]$

Como Ntbs é sempre igual a quatro (4) conforme observar-se na Figura 5, obtém-se:

$\mathrm{Nvbs}=(\mathrm{Ndbp} / 4) \times 100 \quad[\%]$

Aderência de configuração na região ótima devido a PO - (Acop): Este tipo de aderência ocorre quando a seqüência das posições dos três primeiros elementos de PO da empresa participante for exatamente igual à seqüência das posições dos elementos de PO situados na região ótima do MRR.

Aderência de configuração na região boa devido a PO - (Acbp): Este tipo de aderência ocorre quando a seqüência das posições dos quatro últimos elementos de PO da empresa participante for exatamente igual à seqüência das posições dos elementos de PO situados na região boa do MRR.

Aderência de configuração na região ótima devido a SI - (Acos): Este tipo de aderência ocorre quando a seqüência das posições dos dois primeiros componentes de SI da empresa participante for exatamente igual à seqüência das posições dos componentes de SI situados na região ótima do MRR. Como exemplo, observando-se a Figura 5, há ocorrência de Acos para as empresas B e C.

Aderência de configuração na região boa devido a SI - (Acbs): Este tipo de aderência ocorre quando a seqüência das posições dos quatro últimos componentes de SI da 
empresa participante for exatamente igual à seqüência das posições dos componentes de SI situados na região boa do MRR. Como exemplo, observando-se a Figura 5, há ocorrência de Acbs para a empresa C.

Com o objetivo de aferir a competitividade das quatro empresas analisadas aplicaram-se os conceitos expostos acima e através de cálculos determinaram-se os níveis de aderência de cada empresa conforme mostra a Tabela 6 (FERREIRA, 2002).

Tabela 6 - Níveis de aderência nas regiões ótima e boa devido a PO e SI

das empresas participantes

\begin{tabular}{|c|c|c|c|c|}
\hline $\begin{array}{c}\text { Niveis de } \\
\text { Aderência }\end{array}$ & Nvop & Nvbp & Nvos & Nvbs \\
\hline Empresas & {$[\%]$} & {$[\%]$} & {$[\%]$} & {$[\%]$} \\
\hline A e respondente 1 & 66,7 & 75 & 50 & 75 \\
\hline A e respondente 2 & 66,7 & 75 & 0 & 50 \\
\hline B & 66,7 & 75 & 100 e & 100 e \\
\hline C & 66,7 & 75 & $100 \mathrm{e}$ & 100 \\
\hline D & 100 & 100 & 0 & 50 \\
\hline
\end{tabular}

Fonte: FERREIRA, José I.A.X. (2002, p. 177)

A partir dos dados expostos na Tabela 6 calcula-se o nível de aderência $(\mathrm{Nv})$ por empresa, adotando-se como critério a média aritmética dos valores percentuais dos níveis de aderência nas regiões ótima e boa devido a PO e SI. Desta forma, obtêm-se os valores expressos na Tabela 7 (FERREIRA, 2002).

Tabela 7 - Nível de aderência das empresas

\begin{tabular}{|c|c|c|}
\hline Empresas & $\begin{array}{c}\text { Nível de } \\
\text { aderência (Nv) } \\
{[\%]}\end{array}$ & $\begin{array}{c}\text { Ocorrência de } \\
\text { aderência de } \\
\text { configuração }\end{array}$ \\
\hline A & 57 & - \\
\hline B & 85 & Acos e Acbs \\
\hline C & 85 & Acos \\
\hline D & 63 & - \\
\hline
\end{tabular}

Fonte: FERREIRA, José I.A.X. (2002, p. 178) 


\subsection{Comentários}

Analisando o nível de aderência (Nv) e a ocorrência (ou não) de aderência de configuração nas empresas, conforme mostra a Tabela 7, conclui-se que a empresa B está num estado de competitividade melhor que as demais (está mais competitiva) por ter o nível de aderência maior $(\mathrm{Nv}=85 \%)$ em relação as empresas A e $\mathrm{D}$, e possuir também duas aderências de configuração devido a SI, uma na região ótima e outra na região boa, fazendo com que ela (empresa B) esteja também num estado de competitividade melhor que a empresa C. Esta possui apenas uma aderência de configuração devido a SI (região ótima), embora seu nível de aderência $(\mathrm{Nv}=85 \%)$ seja igual ao da empresa $\mathrm{B}$.

Continuando a análise verifica-se que a empresa $\mathrm{D}$, com $\mathrm{Nv}=63 \%$, encontra-se num estado de competitividade melhor que a empresa $\mathrm{A}$, com $\mathrm{Nv}=57 \%$. Verifica-se também que o estado de competitividade das empresas $\mathrm{B}$ e $\mathrm{C}(\mathrm{Nv}=85 \%)$ é melhor do que a empresa $\mathrm{D}$ $(\mathrm{Nv}=63 \%)$.

Por outro lado, observando-se as informações contidas na Tabela 6, conclui-se que a empresa D, por possuir maiores níveis de aderência com relação a parâmetros organizacionais, encontra-se melhor situada em termos competitivos comparativamente às outras empresas, no que diz respeito a estes parâmetros.

Conclui-se também que com relação a sistemas de informação a empresa B encontrase melhor situada, em termos competitivos, do que as outras empresas. O motivo é que a empresa B possui os maiores níveis de aderência devido a SI e possui também duas ocorrências de aderência de configuração devido a SI (regiões ótima e boa).

\section{CONSIDERAÇÕES FINAIS}

Neste trabalho discutiu-se a estrutura de um modelo para aferição da competitividade constituído dos componentes: padrões de competitividade, parâmetros organizacionais, sistemas de informações e medidas de desempenho. Discutiu-se também o modelo de referência reduzido (MRR). Procedeu-se a aplicação deste modelo em quatro empresas através da utilização de um questionário, e comparou-se o resultado obtido com o modelo de referência (reduzido). Desta forma, determinou-se o estado de competitividade das empresas participantes, através da obtenção dos valores dos níveis de aderência, com ocorrência ou não de aderência de configuração. 
Procurou-se mostrar neste trabalho a importância da competitividade e uma maneira de avaliá-la junto às organizações. Procurou-se mostrar também o papel dos diversos agentes, que podem colaborar para isto, tais como: estratégia organizacional, cultura de aprendizagem, estrutura da organização, sistema de recompensa, aprendizagem com o meio exterior, vários tipos de sistemas de informação, e medidas de desempenho relativas a clientes, processos internos, aprendizado e crescimento.

Fica claro, portanto, que a competitividade depende de uma série de fatores interrelacionados que devem ser conduzidos de maneira eficaz para que ela possa ser obtida em sua plenitude.

\section{REFERÊNCIAS}

AGOSTINHO, O.L. Sistemas de Manufatura. Faculdade de Engenharia Mecânica. Campinas: UNICAMP, 2001.

AGOSTINHO, O.L. Sistemas de Informação Aplicados à Estratégia de Manufatura. Faculdade de Engenharia Mecânica. Campinas: UNICAMP, 2002.

BENSAOU, M.; EARL, M. The Right Mind-set for Managing Information Technology. Harvard Business Review, v.76, n.5, p.119-128, Sep.-Oct. 1998.

BUCKLER, B. Practical Steps Towards a Learning Organization: Applying Academic Knowledge to Improvement and Innovation in Business Processes. The Learning Organization - MCB University Press, v.5, n.1, p.15-23, 1998.

CORRÊA, H.L.; GIANESI, I.G.N.; CAON, M. Planejamento, Programação e Controle da Produção: MRP II / ERP. São Paulo: Atlas, 2002. 411p.

DRUCKER, P.F. The Information Executives Truly Need. Harvard Business Review, v.73, n.1, p.54-62, Jan.-Feb. 1995.

FERREIRA, J.I.A.X. Em Direção às Equipes Autogerenciáveis. Revista Controle da Qualidade, v.48, ano 6, p.40-46, Maio 1996.

FERREIRA, J.I.A.X. Proposta de Aferição da Competitividade em Função da Correlação de Parâmetros Organizacionais e de Disponibilização de Informações. Campinas, 2002. 304p.Tese (doutorado) - Faculdade de Engenharia Mecânica, Universidade Estadual de Campinas.

JOHNSTON, R.; BLUMENTRITT, R. Knowledge or Information Differences in Management. Technology Business Review, p.27-31, June-July 2000. 
KAPLAN, R.S.; NORTON, D.P. The Balanced Scorecard: Translating Strategy into Action. Boston: Harvard Business School Press, 2001. 322p.

LAUDON, K.C.; LAUDON, J.P. Management Information Systems: New Approaches to Organization and Technology. Upper Saddle River: Prentice Hall, 2002. 693p.

MANKIN, D.; COHEN, S.G.; BIKSON, T.K. Teams and Technology: Fulfilling the Promise of the New Organization. Boston: Harvard Business School Press, 2002. 284p.

MCGILL, M.E.; SLOCUM, J.W. A Empresa Mais Inteligente. Rio de Janeiro: Campus, 2001. 287p.

NADLER, D.A.; TUSHMAN, M.L. The Organization of the Future: Strategic Imperatives and Core Competences for the $21^{\text {st }}$ Century. IEEE Engineering Management Review, v.27, n. 4, p.96-107, winter 1999.

PORTER, M.E. Estratégia Competitiva. Rio de Janeiro: Campus, 2000. 512p.

PRAHALAD, C.K.; HAMEL, G. Competindo pelo Futuro. Rio de Janeiro: Campus, 2001. 377p.

SENGE, P.M. A Quinta Disciplina: Arte e Prática da Organização que Aprende. São Paulo: Best Seller, 2002.444p.

Artigo recebido em 01/08/2005 e aceito para publicação em 07/08/2006 